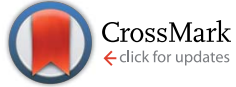

Cite this: RSC Adv., 2015, 5, 34910

DOI: $10.1039 / c 5 r a 90037 a$

www.rsc.org/advances

\title{
Correction: Sustainable construction: admicellar catalysed synthesis of pyrimido[4,5-b]quinolines in an aqueous system
}

\author{
I. R. Siddiqui, ${ }^{* a}$ Pragati Rai, ${ }^{a}$ Rahila, ${ }^{a}$ Hoezyfa Sagir ${ }^{a}$ and Prashant Singh ${ }^{b}$
}

Correction for 'Sustainable construction: admicellar catalysed synthesis of pyrimido[4,5-b]quinolines in an aqueous system' by I. R. Siddiqui et al., RSC Adv., 2015, 5, 27603-27609.

The characterization data of compounds $\mathbf{4 a - 4 1}$ in the Experimental section of the paper is incorrect. The correct characterization data is given below. (Part of this data was previously available as electronic supplementary information (ESI). No additional ESI exists for this work.)

\section{5-(4-Chlorophenyl)benzo[G]pyrimido[4,5-b]quinoline-2,4,6,11(1h,3h,5h,12h)-tetraone (4a)}

Orange powder; mp $294{ }^{\circ} \mathrm{C}$. IR (KBr) $\left(\nu_{\max } / \mathrm{cm}^{-1}\right): 3315,3244,1726,1651,1596,1543 .{ }^{1} \mathrm{H}$ NMR (400 MHz, DMSO-d 6 ): $\delta_{\mathrm{H}}(\mathrm{ppm}) 5.09$ $(\mathrm{s}, 1 \mathrm{H}, \mathrm{CH}), 7.28\left(\mathrm{~d}, J_{\mathrm{HH}}=8.4 \mathrm{~Hz}, 2 \mathrm{H}\right), 7.37\left(\mathrm{~d}, J_{\mathrm{HH}}=8.5 \mathrm{~Hz}, 2 \mathrm{H}\right), 7.77-8.04(\mathrm{~m}, 4 \mathrm{H}, \mathrm{H}-\mathrm{Ar}), 9.39(\mathrm{~s}, 1 \mathrm{H}, \mathrm{NH}), 10.22(\mathrm{~s}, 1 \mathrm{H}, \mathrm{NH}), 10.95$ (s, 1H, NH). ${ }^{13} \mathrm{C}$ NMR $\left(100 \mathrm{MHz}\right.$, DMSO-d $\left.\mathrm{d}_{6}\right): \delta_{\mathrm{C}}(\mathrm{ppm}) 35.0,90.1,118.9,126.8,126.9,128.7,130.8,131.5,132.6,132.9,134.3,135.8$,

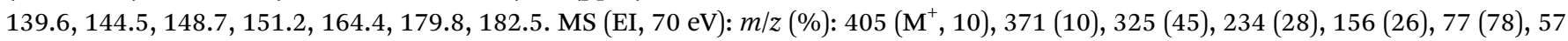
(80), 43 (100). For $\mathrm{C}_{21} \mathrm{H}_{12} \mathrm{ClN}_{3} \mathrm{O}_{4}$ (405.79): C, 62.16; H, 2.98; N, 10.36; found: C, 62.25; H, 2.85; N, 10.49 .

\section{5-(4-Bromophenyl)benzo[ $G]$ pyrimido[4,5-b]quinoline-2,4,6,11(1h,3h,5h,12h)-tetraone (4b)}

Orange powder; mp $299^{\circ} \mathrm{C}$. IR ( $\left.\mathrm{KBr}\right)\left(\nu_{\max } / \mathrm{cm}^{-1}\right): 3406,3249,3054,1735,1661,1605,1577 .{ }^{1} \mathrm{H}$ NMR $\left(400 \mathrm{MHz}, \mathrm{DMSO}-\mathrm{d}_{6}\right): \delta_{\mathrm{H}}(\mathrm{ppm})$ $5.14(\mathrm{~s}, 1 \mathrm{H}, \mathrm{CH}), 6.91-7.99(\mathrm{~m}, 8 \mathrm{H}, \mathrm{H}-\mathrm{Ar}), 9.34(\mathrm{~s}, 1 \mathrm{H}, \mathrm{NH}), 10.18(\mathrm{~s}, 1 \mathrm{H}, \mathrm{NH}), 10.86(\mathrm{~s}, 1 \mathrm{H}, \mathrm{NH}) .{ }^{13} \mathrm{C}$ NMR $\left(100 \mathrm{MHz}, \mathrm{DMSO}-\mathrm{d}_{6}\right): \delta_{\mathrm{C}}$ (ppm) 36.7, 87.9, 119.8, 125.4, 126.8, 126.9, 129.9, 131.6, 132.3, 134.7, 135.9, 138.8, 149.1, 154.8, 159.3, 163.9, 181.4, 184.7. MS (EI, $70 \mathrm{eV}$ ): $m / z$ (\%): 449 (M+1 8), 371 (12), 373 (50), 278 (30), 156 (22), 76 (80), 57 (80), 43 (100). For $\mathrm{C}_{21} \mathrm{H}_{12} \mathrm{BrN}_{3} \mathrm{O}_{4}(450.24): \mathrm{C}, 56.02 ; \mathrm{H}$, 2.69; N, 9.33; found: C, 56.19; H, 2.57; N, 14.34 .

\section{5-(4-Nitrophenyl)benzo[G]pyrimido[4,5-b]quinoline-2,4,6,11(1h,3h,5h,12h)-tetraone (4c)}

Red powder; mp $297^{\circ} \mathrm{C}$. IR (KBr) $\left(\nu_{\max } / \mathrm{cm}^{-1}\right): 3358,3240,3073,1719,1684,1631,1577 .{ }^{1} \mathrm{H}$ NMR $\left(400 \mathrm{MHz}\right.$, DMSO-d 6 ): $\delta_{\mathrm{H}}(\mathrm{ppm})$ $5.25(\mathrm{~s}, 1 \mathrm{H}, \mathrm{CH}), 7.36\left(\mathrm{~d}, J_{\mathrm{HH}}=8.5 \mathrm{~Hz}, 2 \mathrm{H}\right), 7.48\left(\mathrm{~d}, J_{\mathrm{HH}}=8.5 \mathrm{~Hz}, 2 \mathrm{H}\right), 7.73-8.14(\mathrm{~m}, 4 \mathrm{H}, \mathrm{H}-\mathrm{Ar}), 9.31(\mathrm{~s}, 1 \mathrm{H}, \mathrm{NH}), 10.20(\mathrm{~s}, 1 \mathrm{H}, \mathrm{NH})$, $10.83(\mathrm{~s}, 1 \mathrm{H}, \mathrm{NH}) .{ }^{13} \mathrm{C}$ NMR $\left(100 \mathrm{MHz}, \mathrm{DMSO}-\mathrm{d}_{6}\right): \delta_{\mathrm{C}}(\mathrm{ppm}) 37.2,88.0,119.3,126.8,126.9,128.7,131.0,131.2,132.1,132.8,134.7$,

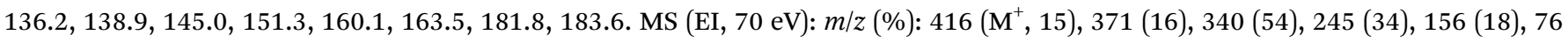
(84), 57 (76), 43 (100). For $\mathrm{C}_{21} \mathrm{H}_{12} \mathrm{~N}_{4} \mathrm{O}_{6}$ (416.34): C, 60.58; H, 2.91; N, 13.46; found: C, 60.72; H, 2.85; N, 13.51.

\section{5-Phenylbenzo[G]pyrimido[4,5-b]quinoline-2,4,6,11(1h,3h,5h,12h)-tetraone (4d)}

Red powder; mp $300{ }^{\circ} \mathrm{C}$. IR (KBr) $\left(\nu_{\max } / \mathrm{cm}^{-1}\right): 3411,3250,3065,1717,1645,1608,1561 .{ }^{1} \mathrm{H}$ NMR $\left(400 \mathrm{MHz}, \mathrm{DMSO}-\mathrm{d}_{6}\right): \delta_{\mathrm{H}}(\mathrm{ppm})$ $5.22(\mathrm{~s}, 1 \mathrm{H}, \mathrm{CH}), 7.11-8.11(\mathrm{~m}, 9 \mathrm{H}, \mathrm{H}-\mathrm{Ar}), 9.29(\mathrm{~s}, 1 \mathrm{H}, \mathrm{NH}), 10.11(\mathrm{~s}, 1 \mathrm{H}, \mathrm{NH}), 10.82(\mathrm{~s}, 1 \mathrm{H}, \mathrm{NH}) .{ }^{13} \mathrm{C}$ NMR $\left(100 \mathrm{MHz}, \mathrm{DMSO}_{\mathrm{C}}\right): \delta_{\mathrm{C}}$ (ppm) 35.6, 86.7, 119.6, 124.4, 126.6, 126.8, 127.9, 128.9, 131.6, 132.4, 134.4, 135.7, 139.8, 151.2, 155.7, 159.3, 163.4, 181.8, 185.6. MS (EI, $70 \mathrm{eV}$ ): $m / z$ (\%): $371\left(\mathrm{M}^{+}, 5\right), 328$ (12), 295 (42), 200 (30), 156 (22), 76 (80), 43 (100). For $\mathrm{C}_{21} \mathrm{H}_{13} \mathrm{~N}_{3} \mathrm{O}_{4}(371.35): \mathrm{C}, 67.92 ; \mathrm{H}, 3.53 ; \mathrm{N}$, 11.32; found: C, 68.06; H, 3.51; N, 11.46. 
5-m-Tolylbenzo[G]pyrimido[4,5-b]quinoline-2,4,6,11(1h,3h,5h,12h)-tetraone (4e)

Red powder; mp $282{ }^{\circ} \mathrm{C}$. IR $(\mathrm{KBr})\left(\nu_{\max } / \mathrm{cm}^{-1}\right): 3327,3268,3050,1723,1657,1610,1529 .{ }^{1} \mathrm{H} \mathrm{NMR}\left(400 \mathrm{MHz}, \mathrm{DMSO}-\mathrm{d}_{6}\right): \delta_{\mathrm{H}}(\mathrm{ppm})$ 2.71 (s, 3H, Me), 5.25 (s, 1H, CH), 7.03-8.15 (m, 8H, H-Ar), 9.24 (s, 1H, NH), 10.16 (s, 1H, NH), 10.89 (s, $1 \mathrm{H}, \mathrm{NH}) .{ }^{13} \mathrm{C} \mathrm{NMR}(100 \mathrm{MHz}$, DMSO-d $\left.{ }_{6}\right): \delta_{\mathrm{C}}(\mathrm{ppm}) 24.5,35.5,86.6,117.6,124.0,126.4,127.0,127.4,128.0,128.7,131.4,132.5,134.1,135.4,135.7,138.1,139.9$, 152.5, 161.2, 163.3, 182.9, 183.8. MS (EI, $70 \mathrm{eV}): \mathrm{m} / z$ (\%): 385 (M $\left.\mathrm{M}^{+}, 8\right), 294$ (40), 214 (22), 156 (28), 91 (70), 57 (78), 43 (100). For $\mathrm{C}_{22} \mathrm{H}_{15} \mathrm{~N}_{3} \mathrm{O}_{4}$ (385.37): C, 68.57; H, 3.92; N, 10.90; found: C, 68.71; H, 3.86; N, 10.85 .

\section{5-o-Tolylbenzo[ $[G]$ pyrimido $[4,5-b]$ quinoline-2,4,6,11(1h,3h,5h,12h)-tetraone $(4 \mathrm{f})$}

Red powder; $\operatorname{mp} 277^{\circ} \mathrm{C}$. IR (KBr) $\left(\nu_{\max } / \mathrm{cm}^{-1}\right): 3417,3248,3055,1714,1657,1609,1533 .{ }^{1} \mathrm{H}$ NMR $\left(400 \mathrm{MHz}, \mathrm{DMSO}-\mathrm{d}_{6}\right): \delta_{\mathrm{H}}(\mathrm{ppm})$ 2.77 (s, 3H, Me), 5.17 (s, 1H, CH), 7.01-8.04 (m, 8H, H-Ar), 9.31 (s, 1H, NH), 10.17 (s, 1H, NH), 10.87 (s, $1 \mathrm{H}, \mathrm{NH}) .{ }^{13} \mathrm{C} \mathrm{NMR}(100 \mathrm{MHz}$, DMSO-d $\left.{ }_{6}\right): \delta_{\mathrm{C}}(\mathrm{ppm}) 22.2,35.6,86.8,117.1,124.0,126.3,126.9,127.5,127.9,128.7,131.3,132.6,134.2,135.3,135.4,138.0,139.1$, 151.2, 158.1, 163.8, 182.4, 183.3. MS (EI, $70 \mathrm{eV}): \mathrm{m} / z$ (\%): 385 (M+1 10$), 309$ (44), 214 (26), 156 (20), 91 (74), 57 (82), 43 (100). For $\mathrm{C}_{22} \mathrm{H}_{15} \mathrm{~N}_{3} \mathrm{O}_{4}$ (385.37): C, 68.57; H, 3.92; N, 10.90; O, 16.61; found: C, 68.80; H, 3.76; N, 10.92 .

\section{5-p-Tolylbenzo $[G]$ pyrimido $[4,5-b]$ quinoline-2,4,6,11(1h,3h,5h,12h)-tetraone $(4 \mathrm{~g})$}

Red powder; mp $31{ }^{\circ} \mathrm{C}$. IR $(\mathrm{KBr})\left(\nu_{\max } / \mathrm{cm}^{-1}\right): 3408,3253,3059,1717,1656,1608,1517 .{ }^{1} \mathrm{H}$ NMR $\left(400 \mathrm{MHz}, \mathrm{DMSO}-\mathrm{d}_{6}\right): \delta_{\mathrm{H}}(\mathrm{ppm})$ $2.19(\mathrm{~s}, 3 \mathrm{H}, \mathrm{Me}), 5.07(\mathrm{~s}, 1 \mathrm{H}, \mathrm{CH}), 7.02\left(\mathrm{~d}, J_{\mathrm{HH}}=7.7 \mathrm{~Hz}, 2 \mathrm{H}\right), 7.19\left(\mathrm{~d}, J_{\mathrm{HH}}=7.7 \mathrm{~Hz}, 2 \mathrm{H}\right), 7.79-8.04(\mathrm{~m}, 4 \mathrm{H}, \mathrm{H}-\mathrm{Ar}), 9.21(\mathrm{~s}, 1 \mathrm{H}, \mathrm{NH})$, 10.14 (s, 1H, NH), 10.89 (s, 1H, NH). ${ }^{13} \mathrm{C}$ NMR (100 MHz, DMSO-d $)_{6}$ : $\delta_{\mathrm{C}}(\mathrm{ppm}) 21.2,35.4,86.5,114.6,126.5,126.8,129.2,130.5$, 130.9, 131.4, 132.5, 134.4, 135.8, 138.7, 144.5, 148.8, 159.1, 163.4, 179.9, 183.2. MS (EI, $70 \mathrm{eV}): \mathrm{m} / z(\%): 385$ (M $\left.{ }^{+}, 5\right), 294(45), 214(30)$, 156 (20), 76 (78), 57 (82), 43 (100). For $\mathrm{C}_{22} \mathrm{H}_{15} \mathrm{~N}_{3} \mathrm{O}_{4}$ (385.37): C, 68.57; H, 3.92; N, 10.90; found: C, 68.51; H, 3.86; N, 10.80.

\section{5-(4-Methoxyphenyl)benzo[G]pyrimido[4,5-b]quinoline-2,4,6,11(1h,3h,5h,12h)-tetraone (4h)}

Orange powder; $\operatorname{mp} 301{ }^{\circ} \mathrm{C}$. IR $(\mathrm{KBr})\left(\nu_{\max } / \mathrm{cm}^{-1}\right): 3423,3178,3057,1704,1681,1611,1533 .{ }^{1} \mathrm{H} \mathrm{NMR}\left(400 \mathrm{MHz}, \mathrm{DMSO}-\mathrm{d}_{6}\right): \delta_{\mathrm{H}}(\mathrm{ppm})$ $3.64(\mathrm{~s}, 3 \mathrm{H}, \mathrm{OMe}), 5.03(\mathrm{~s}, 1 \mathrm{H}, \mathrm{CH}), 6.76\left(\mathrm{~d}, J_{\mathrm{HH}}=7.7 \mathrm{~Hz}, 2 \mathrm{H}, \mathrm{H}-\mathrm{Ar}\right), 7.21\left(\mathrm{~d}, J_{\mathrm{HH}}=7.6 \mathrm{~Hz}, 2 \mathrm{H}, \mathrm{H}-\mathrm{Ar}\right), 7.81-8.02(\mathrm{~m}, 4 \mathrm{H}, \mathrm{H}-\mathrm{Ar}), 9.32(\mathrm{~s}$, $1 \mathrm{H}, \mathrm{NH}), 10.16(\mathrm{~s}, 1 \mathrm{H}, \mathrm{NH}), 10.91(\mathrm{~s}, 1 \mathrm{H}, \mathrm{NH}) .{ }^{13} \mathrm{C}$ NMR $\left(100 \mathrm{MHz}, \mathrm{DMSO}-\mathrm{d}_{6}\right): \delta_{\mathrm{C}}(\mathrm{ppm}) 32.5,56.7,86.5,114.9,124.5,126.2,126.9$, 129.7, 130.0, 130.5, 133.9, 133.1, 133.7, 135.4, 138.0, 144.7, 159.1, 163.6, 179.7, 183.1. MS (EI, $70 \mathrm{eV}): m / z(\%): 401\left(\mathrm{M}^{+}, 15\right), 358(14)$, 325 (30), 230 (26), 156 (22), 57 (76), 43 (100). For $\mathrm{C}_{22} \mathrm{H}_{15} \mathrm{~N}_{3} \mathrm{O}_{5}$ (401.37): C, 65.83; H, 3.77; N, 10.47; found: C, 65.88; H, 3.74; N, 10.58.

\section{5-(4-Chlorophenyl)-1,3-dimethylbenzo[G]pyrimido[4,5-b]quinoline-2,4,6,11(1h,3h,5h,12h)-tetraone $(4 \mathrm{i})$}

Orange powder; mp $251^{\circ} \mathrm{C}$. IR (KBr) $\left(\nu_{\max } / \mathrm{cm}^{-1}\right): 3402,1716,1654,1580,1517 .{ }^{1} \mathrm{H} \mathrm{NMR}\left(400 \mathrm{MHz}, \mathrm{DMSO}-\mathrm{d}_{6}\right): \delta_{\mathrm{H}}(\mathrm{ppm}) 3.19(\mathrm{~s}, 3 \mathrm{H}$, $\mathrm{NMe}), 3.36$ (s, 3H, NMe), 5.66 (s, 1H, CH), 7.10-7.88 (m, 8H, H-Ar), $13.11(\mathrm{~s}, 1 \mathrm{H}, \mathrm{NH}) .{ }^{13} \mathrm{C} \mathrm{NMR}\left(100 \mathrm{MHz}, \mathrm{DMSO}-\mathrm{d}_{6}\right): \delta_{\mathrm{C}}(\mathrm{ppm}) 29.7$, 31.4, 35.3, 86.2, 118.9, 124.0, 126.8, 126.9, 130.4, 131.2, 131.6, 132.6, 134.4, 135.1, 138.9, 150.1, 155.1, 156.4, 164.1, 181.6, 186.9. MS (EI, $70 \mathrm{eV}): m / z(\%): 433\left(\mathrm{M}^{+}, 5\right), 322(8), 271$ (30), 235 (100), $156(72), 76(82), 57$ (26). For $\mathrm{C}_{23} \mathrm{H}_{16} \mathrm{ClN}_{3} \mathrm{O}_{4}(433.84): \mathrm{C}, 63.67 ; \mathrm{H}, 3.72 ; \mathrm{Cl}$, 8.17; N, 9.69;found: C, 63.83; H, 3.54; N, 8.29.

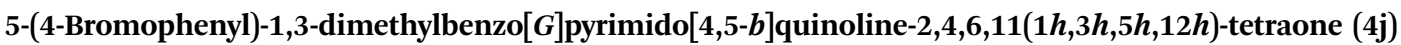

Orange powder; mp $229^{\circ} \mathrm{C}$. IR (KBr) $\left(\nu_{\max } / \mathrm{cm}^{-1}\right): 3398,1705,1656,1575,1511 .{ }^{1} \mathrm{H} \mathrm{NMR}\left(400 \mathrm{MHz}, \mathrm{DMSO}-\mathrm{d}_{6}\right): \delta_{\mathrm{H}}(\mathrm{ppm}) 3.11(\mathrm{~s}, 3 \mathrm{H}$, $\mathrm{NMe}$ ), 3.37 (s, 3H, NMe), 5.81 (s, 1H, CH), 7.18-7.91 (m, 8H, H-Ar), $13.12(\mathrm{~s}, 1 \mathrm{H}, \mathrm{NH}) .{ }^{13} \mathrm{C} \mathrm{NMR}\left(100 \mathrm{MHz}, \mathrm{DMSO}-\mathrm{d}_{6}\right): \delta_{\mathrm{C}}(\mathrm{ppm}) 29.2$, 32.3, 35.3, 89.0, 119.4, 124.1, 126.7, 127.0, 131.0, 131.2, 131.6, 132.4, 134.6, 135.6, 139.1, 151.0, 155.3, 156.4, 164.5, 181.8, 186.7. MS (EI, $70 \mathrm{eV}): m / z(\%): 477$ (M+, 5), 338 (22), 321 (32), 235 (100), 156 (76), 76 (80). For $\mathrm{C}_{23} \mathrm{H}_{16} \mathrm{BrN}_{3} \mathrm{O}_{4}(478.29): \mathrm{C}, 57.76 ; \mathrm{H}, 3.37 ; \mathrm{N}, 8.79$; found: $\mathrm{C}, 57.87 ; \mathrm{H}, 3.36 ; \mathrm{N}, 8.84$.

\section{1,3-Dimethyl-5-p-tolylbenzo[G]pyrimido[4,5-b]quinoline-2,4,6,11(1h,3h,5h,12h)-tetraone (4k)}

Red powder; mp $263{ }^{\circ} \mathrm{C}$. IR (KBr) $\left(\nu_{\max } / \mathrm{cm}^{-1}\right): 3359,1720,1657,1568 .{ }^{1} \mathrm{H}$ NMR $\left(400 \mathrm{MHz}, \mathrm{DMSO}-\mathrm{d}_{6}\right): \delta_{\mathrm{H}}(\mathrm{ppm}) 2.27(\mathrm{~s}, 3 \mathrm{H}, \mathrm{Me}), 3.11$ (s, 3H, NMe), 3.33 (s, 3H, NMe), 5.52 (s, 1H, CH), 6.99 (d, $\left.J_{\mathrm{HH}}=7.8 \mathrm{~Hz}, \mathrm{H}-\mathrm{Ar}\right), 7.21$ (d, $\left.J_{\mathrm{HH}}=7.9 \mathrm{~Hz}, \mathrm{H}-\mathrm{Ar}\right), 7.77-8.06$ (m, 4H, H-Ar), $9.04(\mathrm{~s}, 1 \mathrm{H}, \mathrm{NH}) .{ }^{13} \mathrm{C}$ NMR $\left(100 \mathrm{MHz}, \mathrm{DMSO}-\mathrm{d}_{6}\right): \delta_{\mathrm{C}}(\mathrm{ppm}) 22.8,28.9,31.7,36.6,88.7,116.1,124.4,126.4,126.8,128.9,130.8,131.5$, 134.5, 135.5135.7, 136.9, 139.4, 151.1, 158.1, 163.4, 181.7, 184.6. MS (EI, $70 \mathrm{eV}): \mathrm{m} / z$ (\%): 413 (M+1, 10$), 322$ (6), 275 (20), 235 (100), 156 (72), 76 (80). For $\mathrm{C}_{24} \mathrm{H}_{19} \mathrm{~N}_{3} \mathrm{O}_{4}$ (413.43): C, 69.72; H, 4.63; N, 10.16; found: C, 69.79; H, 4.46; N, 10.20.

\section{1,3-Dimethyl-5-m-tolylbenzo[G]pyrimido[4,5-b]quinoline-2,4,6,11(1h,3h,5h,12h)-tetraone $(41)$}

Red powder; mp $195{ }^{\circ} \mathrm{C}$. IR (KBr) $\left(\nu_{\max } / \mathrm{cm}^{-1}\right): 3327,1705,1656,1569,1529 .{ }^{1} \mathrm{H}$ NMR $\left(400 \mathrm{MHz}, \mathrm{DMSO}-\mathrm{d}_{6}\right): \delta_{\mathrm{H}}(\mathrm{ppm}) 2.24(\mathrm{~s}, 3 \mathrm{H}$, $\mathrm{Me}$ ), 3.17 (s, 3H, NMe), 3.38 (s, 3H, NMe), 5.82 (s, 1H, CH), 6.94-8.01 (m, 8H, H-Ar), 13.02 (s, 1H, NH). ${ }^{13} \mathrm{C} \mathrm{NMR}(100 \mathrm{MHz}, \mathrm{DMSO}-$ $\left.\mathrm{d}_{6}\right): \delta_{\mathrm{C}}(\mathrm{ppm}) 22.5,29.9,31.2,35.6,86.8,111.9,124.7,126.6,127.0,127.3,128.2,128.8,131.5,132.7,134.3,135.4,135.3,137.9,139.2$, 
151.2, 155.3, 164.5, 181.8, 184.4. MS (EI, $70 \mathrm{eV}): \mathrm{m} / z(\%): 413\left(\mathrm{M}^{+}, 8\right), 275$ (16), 257 (22), 235 (100). For $\mathrm{C}_{24} \mathrm{H}_{19} \mathrm{~N}_{3} \mathrm{O}_{4}(413.43): \mathrm{C}, 69.72$; H, 4.63; N, 10.16; found: C, 69.85; H, 4.52; N, 10.07 .

The Royal Society of Chemistry apologises for these errors and any consequent inconvenience to authors and readers. 